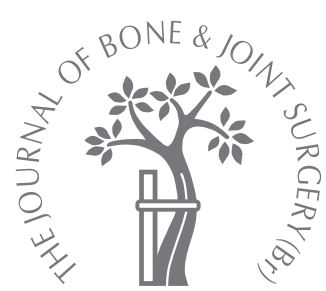

A. L. Armstrong, J. J. Dias

From University Hospitals of Leicester NHS Trust, Leicester, England

A. L. Armstrong,

MBChB(Hons), FRCS(Orth),

Doctor of Medicine, Consultant

Orthopaedic and Trauma

Surgeon

Department of Orthopaedics University Hospitals of

Leicester NHS Trust, Leicester General Hospital, Gwendolen Road, Leicester LE5 4PW, UK.

I. J. Dias, FRCS, MD, Professor of Orthopaedic Surgery

Department of Orthopaedics University Hospitals of

Leicester NHS Trust, Glenfield

Hospital, Groby Road, Leicester LE3 9QP, UK.

Correspondence should be sent to Miss A. L. Armstrong; e-mail: alison@longarms.co.uk

C일 2008 British Editorial Society of Bone and Joint Surgery doi:10.1302/0301-620X.90B5. $20293 \$ 2.00$

$J$ Bone Joint Surg $[\mathrm{Br}]$ 2008;90-B:610-13.

Received 3 October 2007:

Accepted after revision 19 December 2007

\title{
Reconstruction for instability of the sternoclavicular joint using the tendon of the sternocleidomastoid muscle
}

\begin{abstract}
We describe a new method of stabilising a painful unstable sternoclavicular joint using the sternocleidomastoid tendon and passing it through the medial clavicle and onto the manubrium sternum. This method is simple, reproducible and avoids the potential risks of reefing the joint to the first rib. The technique was used in seven cases of sternoclavicular joint instability in six patients who were reviewed at a mean of 39.7 months (15 to 63). Instability was markedly reduced or eliminated in all cases, but in one there was occasional persistant subluxation. There were minor scar complications after two procedures and one patient had transient ulnar neuritis.
\end{abstract}

This procedure provides satisfactory results in the medium term.

Painful instability of the sternoclavicular joint is a rare condition which occurs either after trauma or spontaneously in young women with generalised joint laxity. ${ }^{1-6}$ The instability can be anterior, superior or posterior and may occur acutely or chronically. In acute presentations, stability can be successfully restored with sutures through the bone, bone anchors ${ }^{7,8}$ or other techniques. ${ }^{9-11}$

Surgical stabilisation of the recurrently unstable joint is difficult. Traditionally it has principally involved the use of periosteum or subclavius to secure the clavicle to the first rib with all the attendant risks and variable results. ${ }^{1,6}$ Rockwood and Odor ${ }^{12}$ concluded that patients with instability of spontaneous onset did better without an operation.

We present the medium-term results of a series of seven cases in which the sternocleidomastoid tendon was used to stabilise the sternoclavicular joint.

\section{Patients and Methods}

Between 2000 and 2005, six patients with seven unstable sternoclavicular joints were treated operatively. There were three men and three women with a mean age of 20.7 years (18 to 24). The right shoulder was affected in five patients and the left in two. Two patients had generalised ligamentous laxity. There was a history of trauma in four while in two without ligamentous laxity a precise reason for the instability could not be established. Symptoms had been present for between two and eight years in five shoulders. In one shoulder the symptoms had been present for three months and in one the duration of symptoms had not been recorded. In six of the seven shoulders the instability was painful. Only the patient with the three-month history had painless instability. In this patient the indication for stabilisation was exceptional and was undertaken because the degree of instability prevented him from playing rugby. The instability was apparent on abducting or flexing the arm, and in six cases the patient could dislocate the clavicle and relocate it with a painful clunk.

The symptoms interfered with daily activities such as dressing, especially when they had to put on items which needed to be pulled over the head. Certain activities which required the arm to be circumducted, such as serving at tennis or in some cases simple elevation of the arm, caused a painful clunk.

Instability could be clearly demonstrated with the medial end of the clavicle moving completely or partially out of joint on elevation or circumduction and then relocating with a painful clunk. In all cases the dislocation could be reduced. The direction of instability was anterior in all except one shoulder which had anterosuperior instability. The sternoclavicular joint was assessed for swelling, tenderness and crepitus. In addition, these patients also had a detailed examination of the remainder of the shoulder girdle.

During this period both authors saw other patients with acute or chronic instability of this joint. Patients with an occasional click or clunk which was essentially asymptomatic and non- 


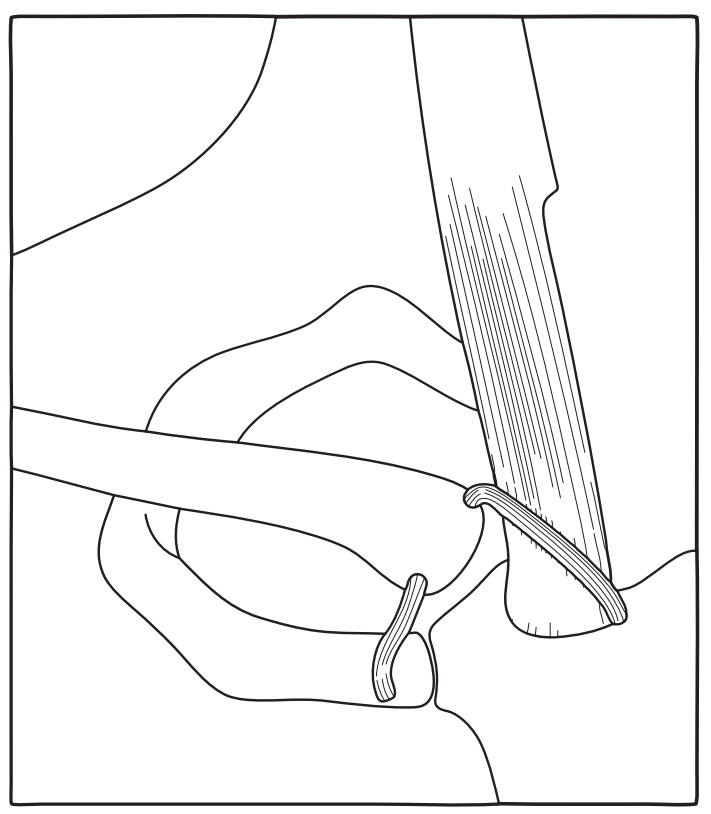

Fig. 1

A diagram demonstrating the harvest of the sternocleidomastoid tendon, kept attached at its sternal end, tubularised and passed through a drill-hole in the medial end of clavicle.

disabling were not offered surgical or other treatment as described by Rockwood and Odor. ${ }^{12}$

Surgical technique. Surgery is undertaken under general anaesthesia in the 'deck chair' position with $30^{\circ}$ of elevation of the head and a pillow under the knees. A $5 \mathrm{~cm}$ transverse incision is centred just below the midline of the medial end of the clavicle and the direction of instability confirmed.

A vertical incision is made in the anterior capsule of the sternoclavicular joint on the sternal side and the state of the intra-articular disc is assessed. If it is ragged, torn or thin and flimsy, it cannot be used to supplement the reconstruction. However, if it is thick and well attached to its deep surface it can be used to attach the capsular sutures at the time of closure.

The proximal skin is mobilised to expose the tendon of the sternal head of the sternocleidomastoid muscle. The quality and anteroposterior thickness of the tendon is assessed to ensure its suitability for transfer. We look for a tendon that is at least $8 \mathrm{~cm}$ in length. This is separated from the muscle and reflected to its sternal attachment but not detached. The tendon is tubularised with a whip stitch of 4.0 vicryl.

A $3.2 \mathrm{~mm}$ drill hole is made from inferior to superior in the medial end of clavicle. The hole is placed $3 \mathrm{~mm}$ to $5 \mathrm{~mm}$ lateral to the attachment of the capsule. The tubularised tendon is passed through the capsule using tendon braiders if possible before being pulled through the drillhole in the clavicle from above to below (Fig. 1). The

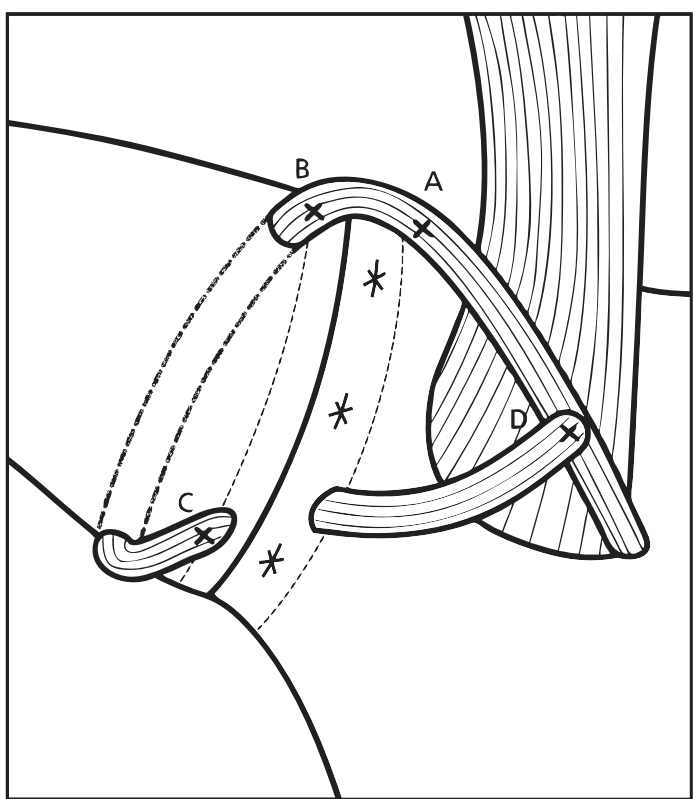

Fig. 2

A diagram demonstrating the method by which the tendon is braided through the anterior capsule to reconstruct the anterior sternoclavicular ligament. Points $A$ to $D$ are the places where the tendon is sutured to the underlying tissue. remaining tendon is braided through the capsule again before being returned to its site of insertion.

The capsule is carefully double-breasted, taking care to pass the sutures through any strong intra-articular disc and through the capsule on the other side. Particular care is taken to advance any intra-articular disc laterally and cephalad and to secure it to the clavicle. Finally, the tendon is pulled taut to reduce the joint and was secured with multiple interrupted 2.0 vicryl sutures (Fig. 2).

The platysma and subcutaneous tissues are closed in layers and the skin closed with an absorbable suture. For the first six weeks post-operatively, the patient's arm is held in a sling and secured to the body with a body belt. No physiotherapy is undertaken at any stage. After six weeks, the patient is allowed to move the arm but advised not to take the elbow behind the mid-lateral line to avoid extension of the shoulder. Normal movement is allowed thereafter. The patient is not allowed to resume most sports until six months post-operatively.

The outcomes assessed included stability, pain and documentation of the state of the scar particularly with regard to any hypertrophy and sensitivity. Complications such as infection, numbness or pain were also documented. We applied a five-point grading of the stability of the joint as reported by the patients and shown in Table I.

\section{Results}

The mean follow-up was 39.7 months (15 to 63). Two patients reported sensitivity in the scar. This was transient 
Table I. Grading of joint stability

\begin{tabular}{lll}
\hline & & Outcome \\
\cline { 3 - 3 } & Stability of joint & Number of shoulders \\
\hline 1 & Stable for all activities & 2 \\
2 & Stable except for transient subluxation (e.g. coughing) but can do everything they & 4 \\
& want to do & 1 \\
3 & Stable most of the time but restricted activity (e.g. sport) & 0 \\
4 & No better after surgery & 0 \\
5 & Worse & \\
\hline
\end{tabular}

for one patient but persisted for the first post-operative year in the other before resolving. The stability of the joint was improved in each case: two were normal, four experienced only transient subluxation and one had occasional subluxation causing a reduction in activity level (Table I). There was no change in sternocleidomastoid function as a result of this procedure. One patient had transient ulnar nerve paraesthesiae which resolved. The rugby player was able to play satisfactorily.

\section{Discussion}

The sternoclavicular joint is stabilised by the anterior sternoclavicular ligament, the attachment of the intra-articular disc and the costoclavicular ligament. A recent study ${ }^{13}$ involving the serial division of ligaments around the sternoclavicular joints in cadavers showed that division of the costoclavicular ligament alone does not destabilise the joint; division of the anterior sternoclavicular ligament with an intact costoclavicular ligament rendered the joint unstable. Detachment of the intra-articular disc also causes instability. ${ }^{13}$ Dennis, Kummer and Zuckerman ${ }^{14}$ have suggested that the posterior sternoclavicular ligament is stronger than the anterior ligament.

A number of studies have reported the outcome of acute dislocation. ${ }^{7-11}$ Double breasting the anterior capsule and reinforcing it with sutures passed through the bone and fixed either with anchors on one side or through drill holes on both sides has been described. ${ }^{7,8}$ This is probably all that is required in the acute situation. However, our study concentrated on recurrent dislocation which was painful in all but one patient.

Most previous methods of stabilising the recurrently unstable joint have concentrated on securing the medial end of the clavicle to the first rib by a variety of techniques. ${ }^{1,2,5,6}$ Burrows, ${ }^{1}$ in 1951 , described the use of the subclavius muscle and its tendon. The tendon was detached from the undersurface of the clavicle, passed through drill holes in the clavicle from beneath to above and then sutured back to the origin of the muscle on the first rib. Bunnell ${ }^{15}$ and Speed ${ }^{16}$ described the use of fascial slings around the medial aspect of the clavicle and first rib.

Booth and Roper ${ }^{4}$ elevated the sternal head of the sternocleidomastoid with a long sleeve of periosteum from the manubrium where it was detached. This was wound round the first rib and through a drill hole in the clavicle, then secured back to itself.
Eskola et $\mathrm{al}^{6}$ used a free tendon graft from the palmaris longus or plantaris passed through drill holes in the sternum and wrapped round the first rib and clavicle. They also used a fascial loop round the first rib and medial clavicle. Qureshi, Shah and Pruzansky ${ }^{17}$ described using the tendon of semitendinosus to stabilise the sternoclavicular joint in a patient with Ehlers-Danlos syndrome.

Brown ${ }^{3}$ turned down the clavicular head of the sternocleidomastoid muscle across the front of the sternoclavicular joint and transfixed the joint with a Rush pin for six to 12 weeks. Lunseth et $\mathrm{al}^{2}$ used subclavius to stabilise the joint but also immobilised it with a Steinman pin for four to six weeks. They did not report any complications related to the use of Steinman pins. However, Brown ${ }^{3}$ reported three serious complications in ten cases using Rush pins. In two cases the pins broke at the joint and one suffered an injury to the pulmonary artery.

The technique described in this paper involves reconstruction of the anterior sternoclavicular ligament. Spencer and $\mathrm{Kuhn}^{18}$ demonstrated in a cadaver study, that reinforcing the anterior capsule by anterior reconstruction of the ligament through the bone is biomechanically superior to costoclavicular stabilisation with subclavius. If the sternocleidomastoid tendon cannot be used, subclavius can be used with the tendon attached to the clavicle or a free palmaris longus tendon graft. We believe that it is also important to reconstruct the attachment of the intra-articular disc carefully as this increases the stability of the joint.

It is difficult to compare our results with those published previously as most studies do not state whether the patient was suffering from recurrent dislocation or whether the joint was chronically dislocated. Brown ${ }^{3}$ described treatment for an acute injury to the sternoclavicular joint in at least four of his ten cases, as did both Abiddin et $\mathrm{al}^{7}$ and Thomas et $\mathrm{al}^{8}$ in two of their three patients.

It is difficult to establish from the literature how many patients achieved stability of the sternoclavicular joint by the various procedures. ${ }^{1-6}$ The authors usually describe the outcome as satisfactory without clearly stating that the joint was rendered stable. The post-operative stability of the sternoclavicular joint was not clearly described in the study of eight patients reported by Eskola et al. ${ }^{6}$ Five patients had pain at rest and seven had pain on activity which was described as moderate in two. These authors graded their outcome on subjective and objective criteria of 
which displacement was one of five items accounting for two of a maximum 12 points. However, from their scoring system and other information it seems likely that up to five of the patients had partial instability when reviewed. In our study two cases were stable after surgery, four had occasional transient instability which did not affect activity level and one patient had transient subluxation with reduced activity.

Only one of our six patients described pain, but this was a result of scar sensitivity which persisted for a year and then resolved; this compares well with the series from Eskola et al. ${ }^{6}$ Other authors report the general outcome but not the incidence of pain after surgery. ${ }^{1-5}$ The complication rate is high. ${ }^{1-6}$ Even when just the anterior capsule was double-breasted and re-inforced with sutures, 25\% (2 of 8 ) had persistent pain. One patient subsequently underwent exision of the medial clavicle. ${ }^{7}$

The drawbacks of this series are that it is small and only reports the medium-term outcome. We cannot be certain whether the stability conferred by this technique will last but other reports with a follow-up of between one and 12 years are also unable to provide an answer. ${ }^{1-6}$

Based on our experience of stabilising anterior and anterosuperior dislocation of the sternoclavicular joint, we feel able to recommend this technique. We advise patients that surgery will improve stability in most cases but that they could be left with an occasional residual click. We also advise them that the pain associated with their dislocation should resolve. There is a small risk of pain occurring in the scar.

This method of treatment using the sternocleidomastoid tendon to stabilise the sternoclavicular joint is simple, reproducible and avoids the potential risks of reefing the joint to the first rib.
No benefits in any form have been received or will be received from a commercial party related directly or indirectly to the subject of this article.

\section{References}

1. Burrows HJ. Tenodesis of subclavius in the treatment of recurrent dislocation of the sterno-clavicular joint. J Bone Joint Surg [Br] 1951;33-B:240-3.

2. Lunseth PA, Chapman KW, Frankel VH. Surgical treatment of chronic dislocation of the sterno-clavicular joint. J Bone Joint Surg [Br] 1975;57-B:193-6.

3. Brown JE. Anteror sternoclavicular dislocation: a method of repair. Am J Orthop 1961;31:184-9.

4. Booth CM, Roper BA. Chronic dislocation of the sternoclavicular joint and operative repair. Clin Orthop 1979;140:17-20.

5. Barth E, Hagen R. Surgical treatment of dislocations of the sternoclavicular joint. Acta Orthop Scand 1983;54:746-53.

6. Eskola A, Vainionpää S, Vastamäki M, Slätis P, Rokkanen P. Operation for old sternoclavicular dislocation: results in 12 cases. J Bone Joint Surg [Br] 1989;71-B:63-

7. Abiddin Z, Sinopidis C, Grocock CJ, Yin O, Frostick SP. Suture anchors for treatment of sternoclavicular joint instability. J Shoulder Elbow Surg 2006;15:315-18.

8. Thomas DP, Williams PR, Hoddinott HC. A 'safe' surgical technique for stabilisation of the sternoclavicular joint: a cadaveric and clinical study. Ann R Coll Surg Engl 2000;82:432-5.

9. Ferrandez L, Yubero J, Usabiaga J, No L, Martin F. Sternoclavicular dislocation: treatment and complications. Ital J Orthop Traumatol 1988;14:349-55.

10. Battaglia TC, Pannunzio ME, Chhabra AB, Degnan GG. Interposition arthroplasty with bone-tendon allograft: a technique for treatment of the unstable sternoclavicular joint. J Orthop Trauma 2005;19:124-9.

11. Féry A, Sommelet J. Sternoclavicular dislocations: observations on the treatment and results of 49 cases. Int Orthop 1988;12:187-95.

12. Rockwood CA Jr, Odor JM. Spontaneous atraumatic anterior subluxation of the sternoclavicular joint. J Bone Joint Surg [Am] 1989;71-A:1280-8.

13. Jaggard M, Wallace WA. Sterno-clavicular joint stability: clinical observations, biomechanical testing and options for improving surgical stabilisation. J Bone Joint Surg $[B r]$ 2008:89-B(Suppl): in press.

14. Dennis MG, Kummer FJ, Zuckerman JD. Dislocations of the sternoclavicular joint. Bul Hosp Jt Dis 2000;59:153-7.

15. Bunnell S. Surgery of the hand. Philadelphia: JB Lippincott Company, 1944:412

16. Speed JS. In: Speed JS, Smith H eds. Campbell's operative orthopaedics. Second ed. St Louis: CV Mosby Company 1949.

17. Qureshi SA, Shah AK, Pruzansky ME. Using the semitendinosus tendon to stabilize sternoclavicular joints in a patient with Ehlers-Dalos syndrome: a case report. $A m$ J Orthop 2005:34:315-18.

18. Spencer EE, Kuhn JE. Biomechanical analysis of reconstructions for sternoclavicular joint instability. J Bone Joint Surg [Am] 2004;86-A:98-105 